\title{
Evaluation of the Quality of Antenatal Care (ANC) Service at Higher 2 Health Center in Jimma, South West Ethiopia
}

\author{
Tirsit Mehari Abate1, Waju Beyene Salgedo 2* , Negalign Berhanu Bayou ${ }^{2}$ \\ ${ }^{1}$ Federal Ministry of Health, Addis Abeba, Ethiopia \\ ${ }^{2}$ Department of Health Economics, Management and Policy, College of Health Sciences, Jimma University, \\ Jimma, Ethiopia \\ Email: tirsitmehari@gmail.com, ${ }^{*}$ dagimwaju@yahoo.com, negenu@gmail.com
}

Received 26 March 2015; accepted 10 April 2015; published 14 April 2015

Copyright (C) 2015 by authors and OALib.

This work is licensed under the Creative Commons Attribution International License (CC BY). http://creativecommons.org/licenses/by/4.0/

(c) $\underset{\mathrm{EY}}{\text { (i) Open Access }}$

\begin{abstract}
Background: Antenatal care is the care that women receive during pregnancy to ensure a healthy outcome for women and newborns. In spite of its clear importance, such services continued to be important determinants of maternal mortality and morbidity throughout the world. Methods: A case study was conducted in Higher 2 Town Health Center, using client exit interviews of 265 clients who came to the health institution. In addition, 10 observations of client-provider interactions, document reviews of 246 clients' documents selected by systematic random sampling technique, and 5 in-depth interviews of the focal persons were conducted. Quantitative data were analyzed using SPSS for windows (SPSS 16.0). Qualitative data were categorized into major thematic areas and presented as narratives in triangulation with the quantitative results. In each evaluation dimension, indicators were used to judge the quality of antenatal care services. Results: Most of the minimum required basic resources recommended by World Health Organization were available in the study site. But there were incomplete sets of laboratory reagents and shortage of some drugs. Comprehensive history was recorded for $\mathbf{9 3 . 4 \%}$ of clients. Only $34.3 \%$ of clients received prescriptions for iron supplementation. Majority $(94.6 \%)$ of the clients were satisfied with the accommodations of antenatal care service. The compliance of the health care providers towards routine laboratory investigations ranged from $25.4 \%$ to $50.1 \%$. Conclusions: The overall quality of the service was judged as fair (69.5\%). Majority of the clients were satisfied with accommodation of ANC services. However, the availability of laboratory reagents and drugs remained to be challenges. Providers' compliance towards standard basic investigations was also far below World Health Organization recommendations and judged as critical. Thus it is recommended that responsible bodies should strive to improve provision of supplies and compliance of the care providers to step-up the quality of care at president capacity level.
\end{abstract}

"Corresponding author.

How to cite this paper: Abate, T., Salgedo, W.B. and Bayou, N.B. (2015) Evaluation of the Quality of Antenatal Care (ANC) Service at Higher 2 Health Center in Jimma, South West Ethiopia. Open Access Library Journal, 2: e1398.

http://dx.doi.org/10.4236/oalib.1101398 


\title{
Keywords
}

\section{ANC, Jimma, Ethiopia}

\author{
Subject Areas: Women's Health
}

\section{Introduction}

Antenatal care is the care that women receive during pregnancy to ensure healthy outcomes for themselves and their newborns. In spite of a clear importance of maternity care, such services continue to be important determinants of maternal mortality and morbidity throughout the world [1].

Each year millions of women, newborns, and children die from preventable causes in the presence of widely known interventions that could save their lives [2]. There were an estimated 358,000 maternal deaths in the world of which 355,000 (99\%) of occur in developing countries. The Maternal Mortality Rate (MMR) in 2008 was 290 in developing regions in contrast to developed regions of 14. Among developing regions, the adult lifetime risk of maternal death is the highest in Sub-Saharan Africa (1 in 31), followed by Oceania (1 in 110) and South Asia (1 in 120), while in the developed regions, it is 1 in 4300 . The leading causes of maternal mortality in developing regions are hemorrhage and hypertension, which together account for half of all deaths in expectant or new mothers. The vast majority of these deaths are avoidable [3].

In a study conducted in six African countries, a third of all pregnant women experienced illness during pregnancy, of whom 3\% required hospitalization. In Sub-Saharan Africa (SSA), an estimated 900,000 babies die as still births during the last 12 weeks of pregnancy. Babies die before onset of labor of ante partum a still birth that accounts $2 / 3$ of all stillbirths in countries. Newborns are affected by problems during pregnancy including preterm birth \& restricted growth as well as other factors. So, provision of quality of health care and effective referral service to mothers and their children is crucial point in redaction of morbidity \& mortality of these vulnerable groups [4].

Ethiopia has been reported to be one of the six countries that contribute about $50 \%$ of the maternal deaths; and it has maternal mortality ratio of 676 per 100,000 with lifetime risk of 1 in 40 . The country also had 109 underfive children mortality rate with annual reduction of 3.6\% from 1990 to 2008 [1].

The fifth Millennium Development Goal (MDG) that includes a proper care of mothers during pregnancy and delivery including ANC links the women $\&$ their family with the health system and increases a chance of getting skilled health care to improve the survival of mother \& health of babies. Women are more likely to give birth with skilled attendant if they have had at least one ANC visit [4].

Measuring the components of antenatal care is essential for assessing the quality of the services, i.e., client provider interaction, availability of resources, and compliance of the service and clients perception on the service. Most of clients don't get information on about the progress, complication, danger signs, plan for complication \& birth and other home care information. Even low range information provision also has a variation on women's background characteristics, i.e., number of parties, education, residence, etc. [1] [5].

Necessary inputs for ANC service have a great impact on the service quality. EDHS 2011 and other studies showed that there was lack of skilled personnel, \& necessary equipment, supplies and drugs in most health facilities. As a result, there were fewer women who took iron and deworming tablets. The clients also compliant as they face long waiting time and providers' interaction and way of approaching has a problem since they are busy or because of other reasons [1] [6].

Seeking skilled delivery service utilization is linked to ANC that leads to a good outcome of maternal neonatal health. In order to achieve this goal of mothers, program managers and stakeholders want to make sure that the service is implemented with a good quality. On the other hand, ANC service is an entry point for other critical health issues like prevention of mother to child transmission (PMTCT) and family planning. Nevertheless, there was no base line data in the district.

\section{Methods}

The study was conducted in Higher 2 Health Center located in Jimma town, southwest Ethiopia from July 2 to 
August 07/2012. The town is located 350 Kilometers from Addis Ababa, the capital city of Ethiopia. The study covered 4 kebeles (small administrative division) namely Mentina, Hermata-Mentina, Hermata-Merkato and Becho-Borii, under the catchment area of the health center. It has an estimated reproductive age group of 11,625 and pregnant women of 2015. The study was conducted by using formative evaluation approach.

A facility based explanatory case study design was employed using both quantitative and qualitative methods data collection. The focus of the evaluation was on the processes of quality of the service. The program was evaluated using Compliance, availability, and accommodation dimensions.

The study population were selected ANC service providers, ANC program managers, sampled cards of ANC clients in the previous six months and 265 ANC Clients who came to the health center during the study period were included in the study. The sample size was calculated by using single population proportion formula taking the assumption of 50\% proportion, 95\% confidence level and 5\% marginal error. The calculated sample populations for card review were cards. All 265 ANC clients, who appeared during the study period, were included in the exit interview. Ten (10) consecutive client-provider interactions starting from the first ANC client were observed on the first day of data collection. For the in-depth interview, five experts were selected purposively: one ANC provider, one laboratory technician, town health office MCH coordinator and head of health center.

Quantitative data was entered into computer and analyzed using SPSS for windows version 16 . The qualitative data were analyzed manually using thematic and content analysis and results were presented in narrative form.

Ethical clearance was secured from ethical committee of public health and medical Science College of Jimma University. Written permission from the regional and zonal research committee was taken and verbal consent was taken from each study participant during data collection.

\section{Results}

\subsection{Sociodemographic Characteristics of the Study Population}

A total of 265 ANC clients were interviewed. The mean age for respondents was $23 \pm 4.5$ years with the range of 15 to 37 years. Majorities 225 (84.9\%) of them were Muslims and 35 (13.2\%) were Orthodox Christianity followers. Majority 126 (47.5\%) of them were unable to read and write. Ethnically, 187 (70.6\%) of them were Oromo and 25 (9.4\%) were Amhara. All of the respondents were married and most 201 (75.8\%) of them were housewives (Table 1).

\subsection{Availability Dimension}

Infrastructure: Observation was made on ANC room privacy and auditory confidentiality. We identified that there was a separate room for ANC service but not auditory confidential as it shares a door with Expanded Program of Immunization (EPI) unit and always open. There was no separate counseling room, running water and functional weight scale for clients. The waiting area was perceived to be satisfactory by $54 \%$ of clients but the waiting area is small.

Availability of trained man power and guideline: The in-depth interviewees ascertained that there were two providers (one diploma nurse \& 1 diploma midwife) consistently working on ANC but only one of them was working during observation. They also assured that both were trained on Focused Antenatal Care (FANC). There was posted FANC guideline during observation.

The source inventory and expert interview showed that there were fetoscopy, weight scale, and fundal height measurements and all were functional but there was no height scale. Blood pressure (BP) apparatus was there but it also was being by other units too that results in interruption of the services sometimes. ANC drugs were available and there were no stock out in last three months.

The health center had necessary laboratory reagents for ANC clients' tests except Venereal Disease Research Laboratory (VDRL) test reagents. The providers blamed the bureautic purchasing process for its absence. All the needed registers, reporting formats, laboratory slips \& ANC cards were available since the health center prints them by itself.

Regarding to the infection prevention supplies, there was a shortage of gloves. In addition, there was no running water hand washing items in the ANC unit but it has sharp boxes. Finally, the input components were judged based on early set criteria of very good, good, fair \& critical using a judgment matrix to declare quality of the service (Table 2). 
Table 1. Socio demographic characteristics of ANC clients at Higher 2 Health Center, Jimma, Ethiopia, July 2012.

\begin{tabular}{|c|c|c|c|}
\hline & & Frequency & Percent \\
\hline \multirow{5}{*}{ Age category } & $<20$ & 88 & 33.2 \\
\hline & 20 to 24 & 75 & 28.3 \\
\hline & 25 to 29 & 66 & 24.9 \\
\hline & $>30$ & 36 & 13.6 \\
\hline & Total & 265 & 100 \\
\hline \multirow{5}{*}{ Religion } & Muslim & 224 & 84.5 \\
\hline & Orthodox & 35 & 13.2 \\
\hline & Protestant & 4 & 1.5 \\
\hline & Catholic & 2 & 0.8 \\
\hline & Total & 265 & 100.0 \\
\hline \multirow{5}{*}{ Ethnicity } & Oromo & 187 & 70.6 \\
\hline & Amhara & 25 & 9.4 \\
\hline & Yem & 12 & 4.5 \\
\hline & Others & 41 & 15.5 \\
\hline & Total & 265 & 100.0 \\
\hline \multirow{6}{*}{ Educational status } & Un able to read \& write & 128 & 48.3 \\
\hline & Able to read \& write & 6 & 2.3 \\
\hline & Grade 1 to 6 & 79 & 29.8 \\
\hline & Grade 7 to 12 & 48 & 18.1 \\
\hline & University/college & 4 & 1.5 \\
\hline & Total & 265 & 100.0 \\
\hline \multirow{7}{*}{ Occupational status } & Un employed & 1 & 0.4 \\
\hline & Government employee & 7 & 2.6 \\
\hline & Merchant & 52 & 19.6 \\
\hline & House wife & 201 & 75.8 \\
\hline & Daily laborer & 3 & 1.1 \\
\hline & Others & 1 & 0.4 \\
\hline & Total & 265 & 100.0 \\
\hline
\end{tabular}

\subsection{Compliance Dimension}

A total of 242 clients' cards were reviewed and 10 ANC sessions were observed to assess compliance of ANC service provision with WHO standards. The inventory of old card showed that the providers had taken personal history of 229 (94.6\%) of the clients, past obstetrics history of 228 (94.2\%) of the clients, medical history of 227 (93.8\%) and surgical history of 228 (94.2\%). 10 clients' examination processes were observed and all were found to be adequate. The four routine physical examinations were recorded on the ANC clients card that included weight for 201 (83.1\%), BP for 164 (67.8\%), fundal height for 184 (67\%) and pallor 227 (93.8\%) of the clients (Table 2). The observation result showed that the three routine tasks (weight, $\mathrm{B} / \mathrm{P}$ and pallor) were carried out at appropriate level but uterine height was done on nine of clients while it should done for all ten. 
Table 2. Judgment and analysis matrix for availability of resources, Higher 2 Health Center, Jimma, July 2012.

\begin{tabular}{|c|c|c|c|c|c|c|c|}
\hline \multicolumn{8}{|c|}{ Resource availability } \\
\hline S. No. & Indicators & Required & Observed & $\begin{array}{l}\text { Agreed } \\
\text { weight }\end{array}$ & Finding & $\%$ & $\begin{array}{l}\text { Judgment } \\
\text { parameter }\end{array}$ \\
\hline 1 & $\begin{array}{l}\text { Availability of physical space providing } \\
\text { ANC ensuring privacy of clients }\end{array}$ & 3 & 2 & 5 & 3.3 & 66 & \multirow{6}{*}{$\begin{array}{l}\geq 85 \% \text { = very good } \\
75 \%-84 \%=\text { good } \\
55 \%-74 \%=\text { fair } \\
\leq 54 \%=\text { critical }\end{array}$} \\
\hline 2 & $\begin{array}{l}\text { Availability of required drugs and } \\
\text { equipment without stock out for last three } \\
\text { months (folic acid, BP apparatus, } \\
\text { fetoscopy, reagents and other) }\end{array}$ & 9 & 4 & 6 & 2.6 & 43.3 & \\
\hline 3 & $\begin{array}{l}\text { Availability of ANC guideline at ANC } \\
\text { room }\end{array}$ & 1 & 1 & 6 & 6 & 100 & \\
\hline 4 & Availability reporting \& recording format & 4 & 4 & 5 & 5 & 100 & \\
\hline \multirow[t]{2}{*}{5} & Number of trained personnel on FANC & 2 & 1 & 8 & 4 & 50 & \\
\hline & Total & & & 30 & 20.9 & 71.9 & \\
\hline
\end{tabular}

Regarding to the routine laboratory Investigation, from the five routine laboratory investigations (urinalysis, VDRL, Hemoglobin, HIV test, blood group and Rh) expected to be carried out according to the standard for each client, only 78 (32.2\%) got hemoglobin test, 85 (35.1\%) got blood group and RH test, 75 (31\%) got VDRL test, 92 (38\%) got counseled for HIV, 72 (25.4\%) got tested for HIV and $126(52.1 \%)$ had got urine test. Among the ten observed first visit clients, 8 were tested for hemoglobin, nine of got HIV test, 8 got tested for VDRL and 6 got test for blood group and Rh. Findings from expert interview disclosed that there was stock out of reagents for VDRL and blood group test in the previous three months but the providers order the tests for clients to get from private clinics. The clients' card review showed that blood test for syphilis was provided only 75 (31\%) of the clients. A laboratory technician declared "We had no reagents for syphilis test in the past three months" (Table 3).

\subsection{Prevention of Diseases and Promotion of Health}

The clients' cards review revealed that only 83 (34.3\%) of clients received prescription for iron supplementation for prevention of anemia, 190 (78.5\%) of clients were referred for TT (Tetanus Toxoid) vaccination. Among the exit interviewees, 179 (74\%) of clients responded that they were using Insecticide Treated Bed net (ITNs). Results from client-provider interaction observations revealed that nine clients were referred for tetanus toxoid vaccination while none of them received prescription for iron supplementation. Findings from resource inventory and expert interviews showed that there was no stock out of the iron/folic acid during the previous six months but providers mostly prescribed iron/folic acid for pregnant women whose hemoglobin level are less than ten and those who have low blood pressure (Table 3).

\subsection{Client Education and Counseling on Nutrition and Danger Sign of Pregnancy}

WHO standard recommends eight items of advice for pregnant women during their visit. But in our observation, from the ten client-provider interactions all received counseling on PMTCT (Prevention of Mother to Child Transmission) while two of them received only counseling on nutrition, three of them on danger signs and none of the clients got counseling on the use of ITNs, hygiene and FP (Family Planning). A health provider responded that "ANC services are provided only by one person and the practicing students who came without a supervisors and mostly miss recording of services provided on clients' cards" (Table 3).

\subsection{Birth Preparedness and Complication Readiness (Danger Sign of Pregnancy)}

Only 79 (32.6\%) and 83 (34.3\%) of the clients received counseling on birth preparedness and danger signs of pregnancy and complication readiness respectively. From 10 clients-provider interactions observed, none got the required counseling about the danger signs. A focal person for ANC said "We usually counsel clients on danger signs if we find that clients have a risk of the danger signs and we mostly don't record on the clients' card but only put 'danger sign or no danger sign'” (Table 3). 
Table 3. Summary of judgment and analysis matrix for compliance of the service, Higher 2 Health Center, Jimma, July 2012.

\begin{tabular}{|c|c|c|c|c|c|c|}
\hline \multicolumn{2}{|c|}{ S. No. Indicators } & \multirow{2}{*}{$\begin{array}{c}\text { Required } \\
4 \\
4\end{array}$} & \multirow{2}{*}{$\begin{array}{c}\text { Weight given } \\
4\end{array}$} & \multirow{2}{*}{$\begin{array}{c}\text { Achieved } \\
3.7\end{array}$} & \multirow{2}{*}{$\begin{array}{c}\% \\
93.4\end{array}$} & \multirow{2}{*}{ Judgment parameters } \\
\hline 1 & $\begin{array}{l}\text { Proportion of clients comprehensive history recorded on } \\
\text { clients' card }\end{array}$ & & & & & \\
\hline 2 & Proportion of clients got routine physical examination & 4 & 4 & 2.9 & 77.9 & \multirow{11}{*}{$\begin{array}{c}\geq 85 \%=\text { very good } \\
75 \%-84 \%=\text { good } \\
55 \%-74 \%=\text { fair } \\
\leq 54 \%=\text { critical }\end{array}$} \\
\hline 3 & Proportion of clients who got blood test for grouping \& Rh & 1 & 2 & 0.7 & 35.1 & \\
\hline 4 & Proportion of clients who got blood test for hgb & 1 & 2 & 0.6 & 32.2 & \\
\hline 5 & Proportion of clients who got blood test for VDRL & 1 & 2 & 0.6 & 31 & \\
\hline 6 & Proportion of clients who got urine test & 1 & 4 & 2 & 52.1 & \\
\hline 7 & Proportion of clients who got TT & 1 & 4 & 3.1 & 78.5 & \\
\hline 8 & Proportion of clients who got iron tablet & 1 & 4 & 1.4 & 34.3 & \\
\hline 9 & $\begin{array}{l}\text { Proportion of clients who got information on danger sign, } \\
\text { birth preparedness \& complication readiness plan etc. }\end{array}$ & 2 & 6 & 2 & 33.4 & \\
\hline 10 & Proportion of clients who got pre-test counselling for HIV & 1 & 4 & 1.5 & 38 & \\
\hline \multirow[t]{2}{*}{11} & Proportion of clients who got tested for HIV & 1 & 4 & 1 & 25.4 & \\
\hline & Total & 14 & 40 & 19.5 & 48.3 & \\
\hline
\end{tabular}

\subsection{Accommodation of ANC Structure and Services}

A total of 265 ANC clients were interviewed soon after their completion of the day's service. The mean age of the respondents was 23 years. Majority, 225 (84.9\%), of them were Muslims followed by Orthodox (13.2\%). 126 (47.5\%) of them were unable to read and write. Ethnically, 187 (70.6\%) were Oromo followed by Amhara (9.4\%). All were married and most of them were 201 (75.8\%) were housewives. 74 (27.9\%) of clients were first visit, 116 (43.8\%) were second visit, 52 (19.6\%) were third visit, 21 (7.9\%) were fourth visit and $2(0.8 \%)$ of the clients were above fourth visit (Table 3).

The result indicated that 224 (84.5\%) of the respondents were satisfied with adequacy of working hours and 229 (86.4\%) were satisfied with the time spent with the provider. All (100\%) of them perceived that the appointing system for follow-up is convenient while 143 (54\%) were satisfied with the convenience of waiting area. The clients ascertained that the average waiting time spent for routine antenatal follow-up to see a service provider was 29 minutes but 133 (50.2\%) of the respondents waited for less than 29 minutes. Majority, 185 (69.8\%) said that the consultation time with a provider was for less than 15 minutes and only $80(30.2 \%)$ said that it was greater than 15 minutes (Table 4).

The overall quality of ANC services was judged by taking the composite average of quality of care indicated above in each dimension surveyed (Table 5).

\section{Discussions}

The results of this evaluation revealed that the study health center fulfilled most of required equipments for ANC according to WHO focused ANC standard with shortage of some supplies and laboratory reagents. Absence of stock-out of drug supplies is another good finding in this study. This result is different from the study conducted in rural Tanzania that showed all health units experienced stock-outs of drugs and equipment where only five out of the eight health units had a well-functioning blood pressure machine and most health units lacked iron tablets. The differences might be related to difference of the setup and methods used [7].

Similarly the adequacy of trained man power those were trained on the FANC in our study is different from the study done in the four selected (RCH) clinics where only one out of the three care providers had been trained on the FANC guidelines [8]. This difference might be related to differences in setup and methods of study.

The focused ANC guideline recommends counseling room with doors and windows to ensure auditory and visual privacy for ANC clients but our observation showed that the level of auditory and visual privacy of ANC/PMTCT counseling room not secured with door and this compromises patient confidentiality and personal dignity. 
Table 4. Matrix of judgment and analysis of accommodation, Higher 2 Health Center, Jimma, July 2012.

\begin{tabular}{|c|c|c|c|c|c|}
\hline $\mathrm{S} / \mathrm{N}$ & Indicators & Weight given & Achieved & $\%$ & Judgment parameter \\
\hline 1 & $\%$ of ANC clients who perceived that waiting area is convenient & 5 & 2.7 & 54 & \multirow{7}{*}{$\begin{array}{c}\geq 85 \%=\text { very good } \\
75 \%-84 \%=\text { good } \\
55 \%-74 \%=\text { fair } \\
\leq 54 \%=\text { critical }\end{array}$} \\
\hline 2 & $\%$ of client who satisfied with adequacy of working hours & 5 & 4.3 & 84.5 & \\
\hline 3 & Proportion of clients who are satisfied by their privacy ensured & 5 & 4.6 & 92.5 & \\
\hline 4 & Proportion of client satisfied with waiting time & 5 & 4.3 & 86.4 & \\
\hline 5 & $\begin{array}{l}\text { Proportion of ANC clients who are satisfied with appointment } \\
\text { schedule/time }\end{array}$ & 5 & 5 & 100 & \\
\hline \multirow[t]{2}{*}{6} & Proportion of clients who are satisfied with provider respect & 5 & 4.7 & 94.3 & \\
\hline & Total & 30 & 25.6 & 85.3 & \\
\hline
\end{tabular}

Table 5. Overall quality of Antenatal care service at Higher 2 Health Center by Evaluation Dimensions, Jimma, July 2012.

\begin{tabular}{ccc}
\hline Dimensions & Judgment parameter & Observed quality level \\
\hline Availability (30\%) & & $71.9 \%(20.9)$ \\
Compliance (40\%) & $\geq 85 \%=$ very good & $48.3 \%(19.5)$ \\
Accommodation (30\%) & $75 \%-84 \%=$ good & $85.3 \%(25.6)$ \\
Overall quality of ANC service & $55 \%=74 \%=$ fair & $66.8 \%$ fair \\
\hline
\end{tabular}

The overall recorded Compliance of providers (94.6\%) to the national standard in this study can be taken as a good achievement. This finding is more or less comparable with WHO recommendation. The result also showed that comprehensive history was taken for all clients in 10 client-provider interactions where the four routine physical examinations of clients' weight, BP, urine test and pallor were performed at the level of $83.1 \%, 67.8 \%$, $67 \%$ and $93.8 \%$ respectively. These findings were comparable to other studies conducted in Ethiopia, Gambia and Tanzania which indicated that the similar routine physical examinations were recorded for over $85 \%$ of all clients for BP, weight and uterine height [4] [9] [10].

Hemoglobin test was performed for $32.2 \%$ of clients, 35.1 of clients got blood group and Rh test, 31\% for VDRL, 25.4\% HIV and 52.1\% urine tested were performed. These findings are different from similar study conducted in Tanzania and Gambia which was $9 \%$ in terms of blood and $41 \%$ in terms of urine tests respectively [4] [9] [10]. This difference could be due to variation of supply provision from one to another.

With regard to prevention services on ANC, only 34.3\% of clients received prescription for iron supplementation the reason being the common practice of care providers limiting its prescription to clients with low Hgb level. This finding is better than the EDHS 2011 finding where only 6\% of clients received prescription for iron/folic acid [4] but in contrary to WHO's recommendation that requires all pregnant women to get it prevent the probability of anemia as result high nutrition demand of pregnancy. On the other hand, only 83 (34.3\%) of the clients responded that they identify the danger signs of pregnancy and complication readiness. This finding worse than the experience of Tanzania where $54 \%$ women were able to mention at least one obstetric danger sign requiring medical attention.

Moreover, the observation that none of the 10 clients-provider interactions got counseling about birth preparedness, danger sing and complication readiness in this study puts the situation more worse. A focal person of ANC supported this by saying” we usually counsel clients if we find that clients show danger signs and even we mostly record on the clients' card but put whether clients' got danger sign or not simply. The findings are somewhat different from the study conducted in Gambia and Tanzania which showed greater than $60 \%$ clients declared their understanding of major danger signs pregnancy and child birth [9] [11].

Our study showed that majority (85.3\%) of the clients was satisfied with accommodation of ANC services. This finding is better than study conducted in Hyderabad Sindh which revealed half of the women were satisfied with the overall care provided to them. Majority (86.4\%) of clients satisfied with the mean waiting time of 28 minutes. This is better than the study conducted in Hyderabad Sindh in which about 86.2\% women said that they 
have to wait for more than two hours for checkups [12].

The average consultation time in this study was 15 minutes but majorities, 185 (69.8\%), of clients received consultation from provider in less than 15 minutes. This is somewhat better than the finding of a study done in Gambia where only $2.5 \%$ clients said that they spent 10 or more minutes of consultation with their care providers [12] but lesser than the mean total duration for initial ANC consultations of 40.1 minutes (range 33 - 47) a study done in Tanzania [11].

With regard to privacy of examination rooms, 245 (92.5\%) of clients said that they were satisfied with the privacy ensured but this higher than the finding of a study conducted in Gambia in which about $64 \%$ felt they were attended to with adequate privacy. The difference might be related to the difference of the setups and perception of the clients.

\section{Conclusions}

This evaluation study revealed that the overall availability of the required resources recorded as $74.6 \%$ of the national standard was judged as fair according to preset judgment parameter. Majority clients were satisfied by accommodations of focused ANC service. However, provider compliance level to the key components of WHO focused ANC was far below the WHO recommendations and evaluation criteria.

Thus it is recommended that the health center should give attention to improve auditory and visual privacy of ANC clinic, maintain running water in the clinic to ensure infection prevention, conduct regular resource inventory to prevent stock outs and motivate the providers to improve their clinical practice. The responsible body of the town health department should carry out regular supportive supervision in order to maintain the performance of the health center according to the nationally accepted WHO recommendations.

\section{Acknowledgements}

We are grateful to Jimma University for funding our work. We would like to acknowledge stakeholders for their participation \& valuable suggestions during stakeholders' meeting. We would like to thank the study participants for their willingness and cooperation during data collection and the staffs of the health center for their cooperation.

\section{Competing Interests}

There is no competing interest with the presented data as external data collectors collected it. There was not financial interest $\mathrm{b} / \mathrm{n}$ the funder and the research area community and us. We, the researchers, have no any form of competing financial and non-financial interest between ourselves.

\section{Authors' Contributions}

We, the three, had active contribution in the proposal development, defending for fund obtaining, data collection and data analysis and manuscript preparation process of this work.

\section{References}

[1] Central Statistical Agency [Ethiopia] and ICF International (2012) Ethiopia Demographic and Health Survey 2011. Central Statistical Agency and ICF International, Addis Ababa, Ethiopia and Calverton, Maryland, USA. http://www.measuredhs.com

[2] Erin, S., Tinker, A. and Ruben, J. (2006) The Maternal-Newborn-Child Health Continuum of Care. A Collective Effort to Save Lives. www.savethechildren.org

[3] WHO, UNICEF, UNFPA \& World Bank (2008) Trends in Maternal Mortality. 1990 to 2010. www.who.int

[4] Lincetto, O., Mothebesoane-Anoh, S., Gomez, P. and Munjanja, S. (2012) Opportunities for Africa's Newborns. Antenatal Care. www.who.int/entity/pmnch/media/publications/aonsectionIII_2.pdf

[5] Pembe, A.B., Urassa, D.P., Carlstedt, A., Lindmark, G., Nyström, L. and Darj, E. (2012) Rural Tanzanian Women’s Awareness of Danger Signs of Obstetric Complications. BMC Pregnancy and Childbirth, 9, 12. http://www.biomedcentral.com/1471-2393/9/12

[6] (2007) Disease Control Project Priorities. Maternal Deaths, Very Good Lack of Progress. http://www.dcp2.org 
[7] Donabedian, A. (2005) Evaluating the Quality of Medical Care. Milbank Quarterly, 83, 691-729. http://onlinelibrary.wiley.com/doi/10.1111/j.1468-0009.2005.00397.x/abstract

[8] Gross, K., et al. (2012) Antenatal Care in Practice: An Exploratory Study in Antenatal Care Clinics. BMC Pregnancy and Childbirth, 11, 36. http://www.biomedcentral.com/1471-2393/11/36

[9] Anya, S.E., Hydara, A. and Jaiteh, L.E.S. (2008) Antenatal Care in the Gambia: Missed Opportunity for Information, Education and Communication. BMC Pregnancy and Childbirth, 8, 9. www.biomedcentral.com/1471-2393/8/9/

[10] Sarker, M., Schmid, G., Larsson, E., Kirenga, S., De Allegri, M., Neuhann, F., Mbunda, T., Lekule, I. and Müller, O. (2010) Quality of Antenatal Care in Rural Southern Tanzania: A Reality Check. BMC Research Notes, 3, 209. www.biomedcentral.com/content/pdf/1756-0500-3-209.pdf

[11] Magoma, M., Requejo, J., Merialdi, M., Campbell, O.M.R., Cousens, S. and Filippia, V. (2011) How Much Time Is Available for Antenatal Care Consultations? Assessment of the Quality of Care in Rural Tanzania. BMC Pregnancy and Childbirth, 11, 64. www.biomedcentral.com/1471-2393/11/64

[12] Nisar, N. and Amjad, R. (2007) Pattern of Antenatal Care Provided at a Public Sector Hospital Hyderabad Sindh. Journal of Ayub Medical College Abbottabad, 19, 11-13. www.ayubmed.edu.pk/

\section{Abbreviations}

ANC: Antenatal Care

MDG: Millennium Development Goal

EDHS: Ethiopian Demographic and Health Survey

EPI: Expanded Program of Immunization

FANC: Focused Antenatal Care

FP: Family Planning

ITN: Insecticide Treated Bed Net

PMTCT: Prevention of Mother to Child Transmission

SSA: Sub-Saharan Africa

TT: Tetanus Toxoid

VDRL: Venereal Disease Research Laboratory 